\title{
AVALIAÇÃO DE PROTOCOLOS DE CLAREAMENTO DENTÁRIO MODIFICADOS POR FONTES LUMINOSAS
}

Evaluation of dental bleaching protocols modified by light sources

Evaluación de protocolos de blanqueamiento dental modificados por fuentes de luz

Thayse Gonzaga Gomes • Universidade Estadual da Paraíba - campus Araruna • Cirurgiã-Dentista • E-mail: thaysegg@hotmail.com

Talina Rocha Balbino dos Santos • Universidade Estadual da Paraíba - campus Araruna • Cirurgiã-Dentista • E-mail: talina_rocha@hotmail.com

Nathália Larissa Bezerra Lima • Universidade de Pernambuco - campus Arcoverde - Acadêmica do Curso de Odontologia • E-mail: nathalialbl.123@gmail.com

Pedro Henrique Sette-de-Souza - Universidade de Pernambuco - campus Arcoverde • Professor do Curso de Odontologia e do Programa de Pós-Graduação em Saúde e Desenvolvimento Socioambiental • E-mail: pedro.souza@upe.br

Autor responsável pela correspondência:

Pedro Henrique Sette-de-Souza • E-mail: pedro.souza@upe.br 


\section{RESUMO}

Introdução: O clareamento dental é o procedimento estético mais realizado nos consultórios odontológicos. O uso de fontes luminosas tem sido recomendado para acelerar a ação do gel clareador, entretanto evidências científicas são controversas no que se diz respeito a essas fontes. Objetivo: Avaliar protocolos de clareamento dentário modificados por fontes luminosas, objetivando verificar, qual a influência dessas fontes no procedimento de clareamento dentário. Metodologia: Sendo essa, uma pesquisa que utilizou material biológico, a mesma foi submetida e aprovada pelo Comitê de Ética em Pesquisa da UEPB. Realizou-se a obtenção de dentes ( $n=20)$ por meio de parceria com o Banco de Dentes Humanos do curso de Odontologia da UEPB Campus VIII. Posteriormente realizou-se identificação dos dentes, em seguida registro inicial da cor do dente (escala VITA), o preparo dos blocos dentários e seleção dos grupos. Os dentes foram submetidos a dois protocolos de clareamentos distintos, com luz halógena e com LED, com ou sem troca do gel. Por fim, realizada a análise estatística. Resultados: A modificação do protocolo, seja quanto o tempo de aplicação do agente clareador, seja quanto à fonte de luz utilizada, alcança resultados satisfatórios. Houve diferença estatisticamente significativa entre os grupos da luz halógena $(p=0.041)$ Conclusões: Tendo em vista os resultados e a discussão encontrados na pesquisa, podemos verificar que as evidências científicas nos levam a observar que não há bases suficientes que comprovem a eficácia e benefícios a curto prazo da modificação dos protocolos de clareamento dental pelas fontes luminosas.

Palavras-Chave: Clareamento dental, Odontologia, Luz, Laser

\section{ABSTRACT}

Introduction: Tooth whitening is the most common cosmetic procedure performed in dental offices. The use of light sources has been recommended to accelerate the action of the whitening gel; however scientific evidence is controversial regarding these sources. Objective: Evaluate tooth whitening protocols modified by light sources, aiming to verify, what is the influence of these sources in the tooth whitening procedure. Methodology: This being a research that used biological material, it was submitted and approved by the Research Ethics Committee of UEPB. Teeth $(n=20)$ were obtained through a partnership with the Human Teeth Bank of the Dentistry course at UEPB Campus VIII. Subsequently, teeth identification was performed, followed by initial registration of tooth color (VITA scale), preparation of dental blocks and selection of groups. The teeth were submitted to two different whitening protocols, with halogen light and with LED, with or without changing the gel. Finally, statistical analysis was performed. Results: The modification of the protocol, both in terms of the application time of the bleaching agent and in terms of the light source ised, achieves satisfactory results. There was statistically significant difference etween groups with halogen light $(p=0.041)$. Conclusions: In view of the results and discussion found in the research, we can see that the scientific evidence leads us to serve that there are not enough bases to prove the effectiveness and short-term nefits of modifying the teeth whitening protocols by light sources.

ywords: Tooth Bleaching, Dentisty, Light, Lasers. 


\section{ciência
piural}

\section{RESUMEN}

Introducción: El blanqueamiento dental es el procedimiento cosmético más común que se realiza en consultorios dentales. Se ha recomendado el uso de fuentes de luz para acelerar la acción del gel blanqueador, sin embargo, la evidencia científica es controvertida con respecto a estas fuentes. Objetivo: Evaluar los protocolos de blanqueamiento dental modificados por fuentes de luz, con el objetivo de verificar cuál es la influencia de estas fuentes en el procedimiento de blanqueamiento dental. Metodología: Al tratarse de una investigación que utilizó material biológico, fue presentada y aprobada por el Comité de Ética en Investigación de la UEPB. Los dientes $(n=20)$ se obtuvieron a través de una asociación con el Banco de Dientes Humanos del curso de Odontología en el Campus VIII de la UEPB. Posteriormente, se realizó la identificación de los dientes, seguida del registro inicial del color del diente (escala VITA), la preparación de los bloques dentales y la selección de grupos. Los dientes fueron sometidos a dos protocolos diferentes de blanqueamiento, con luz halógena y con LED, con o sin cambiar el gel. Finalmente, se realizó un análisis estadístico. Resultados: La modificación del protocolo, tanto en términos del tiempo de aplicación del agente blanqueador como en términos de la fuente de luz utilizada, logra resultados satisfactorios. Hubo diferencias estadísticamente significativas entre los grupos com luz halógena ( $\mathrm{p}=0.041$ ). Conclusiones: En vista de los resultados y la discusión encontrada en la investigación, podemos ver que la evidencia científica nos lleva a observar que no hay suficientes bases para probar la efectividad y los beneficios a corto plazo de modificar los protocolos de blanqueamiento dental por fuentes de luz.

Palabras clave: Blanqueamiento de Dientes, Odontología, Luz, Rayos Láser. 


\section{Introdução}

O conceito de beleza possui uma conotação subjetiva às diferentes culturas existentes nas diferentes épocas e períodos vivenciados pela humanidade. Atualmente, observamos a crescente busca pela estética facial, sobretudo a estética do sorriso, no que se diz respeito principalmente a forma e coloração dos dentes. Esse novo parâmetro estabelecido pelo aumento da demanda em procedimentos estéticos, exige uma Odontologia mais preparada e especializada nesse aspecto. Nesse sentido, têm-se observado que o clareamento dentário vem sendo muito solicitado pelos pacientes que querem um sorriso mais estético e agradável1-3.

A cor do dente é determinada por uma combinação de diferentes propriedades ópticas do esmalte, dentina e da polpa. A descoloração das estruturas dentárias pode variar em etiologia, aparência, localização e gravidade. Além disso, pode ser classificada como intrínseca ou extrínseca. Embora a descoloração extrínseca possa ser removida com um procedimento de limpeza profilática, a coloração intrínseca requer branqueamento químico ${ }^{1-2,4}$.

Desde a introdução de tratamentos clareadores em consultório, o uso de fontes luminosas tem sido recomendada para acelerar a ação do gel de clareamento ${ }^{1,4-5}$. A vantagem teórica que recai sobre a utilização das fontes luminosas, reside na capacidade da luz em aquecer o peróxido de hidrogênio, aumentando a taxa de decomposição de oxigênio para formar radicais livres, aumentando a liberação dos compostos contidos nas manchas ${ }^{4,5}$. Porém é relevante destacar que o aquecimento dos agentes da reação pode causar danos às estruturas dentarias sadias. Sabe-se que temperaturas além de $5,5^{\circ} \mathrm{C}$ da condição normal $\left(36^{\circ} \mathrm{C}\right)$, podem provocar danos irreversíveis aos tecidos, sendo prejudicial principalmente ao tecido pulpar ${ }^{1,5-8}$.

A sensibilidade dentária é um dos efeitos colaterais mais comuns do tratamento lareador. Este fenômeno depende diretamente da concentração do agente clareador e tempo de aplicação. A sensibilidade dentária normalmente persiste por até quatro ias após a conclusão do tratamento clareador. Todavia, períodos mais longos de sibilidade têm sido relatados ${ }^{8,9}$. 
Para os procedimentos realizados com a ativação luminosa, é de grande importância para o resultado final do tratamento, bem como para a satisfação e conforto do paciente, que haja por parte do profissional o domínio à respeito da interação ocorrida entre as fontes de luz e os agentes clareadores, além dos possíveis efeitos dessa interação sobre as estruturas dentárias e se a fonte luminosa utilizada influenciará o resultado final do clareamento. Conforme exposto, o objetivo desse trabalho é avaliar a influência de fontes luminosas no procedimento de clareamento dentário.

\section{Metodologia}

Pesquisa laboratorial submetida à apreciação pelo Comitê de Ética em Pesquisa da UEPB, sendo aprovada com o parecer número 2.431.671. Após isto, os dentes utilizados nessa pesquisa foram doados pelo Banco de Dentes Humanos do curso de Odontologia da UEPB - campus VIII, conforme preconizado pela resolução do Conselho Nacional de Saúde 466/2012. Para esse propósito, 20 molares com coroa hígida foram selecionados, uma vez que a porção coronária foi dividida ao meio, totalizando 40 espécimes ao final.

O preparo dos blocos dentários, bem como a seleção de cor foi baseado na metodologia previamente descrita por Santos et al $(2018)^{10}$. Inicialmente, os dentes selecionados foram codificados em blocos e grupos, e classificados de acordo com a escala VITA® clássica (Zahnfabrik, Sackingen, Alemanha), seguindo metodologia adotada por Polydorou et al. (2013) e Gonçalves et al (2017) 6,11. Para realizar a seleção da cor, dois examinadores independentes passaram por um processo de calibração prévia (kappa $=0,92)$. Durante o preparo, os dentes foram seccionados na junção amelocementária por um disco diamantado para separar a porção coronária da radicular.

Após esse procedimento, realizou-se o sorteio dos blocos por "cara ou coroa", tal modo que "cara" significou grupo 1 ou 3, e "coroa" grupo 2 ou 4 . Se a porção esial de um bloco ficasse no grupo 1 ou 3, a porção distal do mesmo deverá ficar rigatoriamente no grupo 2 ou 4 . Ao término do processo de aleatorização, formouuatro grupos distintos: 
- $\quad$ Grupo 1 = Protocolo convencional + Luz Halógena

- $\quad$ Grupo 2 = Protocolo modificado + Luz Halógena

- $\quad$ Grupo 3 = Protocolo convencional + LED.

- $\quad$ Grupo 4 = Protocolo modificado + LED.

Para este estudo, utilizou-se o gel clareador a base de Peróxido de Hidrogênio na concentração de 35\% (Whiteness HP - FGM® - Joinville, SC, Brasil). O gel foi aplicado na superfície de cada espécime com auxílio de um micropincel, seguindo protocolos distintos:

(I) Convencional = o gel foi aplicado e deixado na superfície por 15 minutos, sendo o espécime lavado com água destilada e algodão. O procedimento foi repetido por três vezes;

(II) Modificado = o gel foi aplicado e deixado na superfície por 45 minutos, sofrendo uma lavagem única ao final do processo, segundo resultados de estudo prévio $^{10}$.

Durante ambos os processos, bolhas que se formavam pela reação do peróxido eram desfeitas com auxílio do mesmo micropincel utilizado para a aplicação do gel. $\mathrm{O}$ aparelho fotopolimerizador com luz halógena utilizado foi o DentLight de lâmpada halógena com refletor 12V, 75W (KOMLux® - Campinas, SP, Brasil), enquanto o LED utilizado possuía $1250 \mathrm{~mW}$ de potência, cedido pela DIACIN/IFRN. Durante todo o protocolo utilizado, os espécimes foram submetidos as luzes. Ao final de todo o processo, os espécimes tiveram novamente a cor aferida.

Para a análise estatística, inicialmente foi aplicado o teste de KolmogorovSmirnov para avaliar a igualdade de distribuições dos resultados das amostras (normalidade). Após isso, lançou-se mão do teste de Kruskall-Wallis (comparação de grupos independentes entre si). Em seguida, realizou-se o teste de Mann-Whitney com correção de Bonferroni para verificar entre quais grupos poderia estar a diferença tatisticamente significativa. Um $\mathrm{p} \leq 0,05$ foi adotado como indicativo de significância atística. 


\section{Resultados}

Os dados alcançados na presente pesquisa foram submetidos à análise estatística. A modificação do protocolo, seja quanto o tempo de aplicação do agente clareador, seja quanto à fonte de luz utilizada, alcança resultados satisfatórios. O efeito clareador mais satisfatório, em termos absolutos, foi alcançado nos grupos em que o gel clareador não foi removido. Contudo, a única diferença estatisticamente significativa entre os grupos foi observada quando se aplicava luz halógena (Tabela $1)$.

Tabela 1: Registro de cores inicial, final e a diferença entre os grupos. Araruna-PB, 2019.

\begin{tabular}{ccccc}
\hline Grupo & Cor Inicial & Cor Final & Diferença & p-valor \\
\hline G1 & $12,00(10,25 / 13,00)$ & $7,00(2,25 / 9,00)$ & $5,00^{\mathrm{a}}(2,25 / 8,75)$ & 0,041 \\
G2 & $12,00(10,25 / 13,00)$ & $3,00(3,00 / 4,50)$ & $9,00^{\mathrm{b}}(6,25 / 10,00)$ & \\
G3 & $10,00(6,00 / 11,75)$ & $5,00(2,25 / 6,75)$ & $4,50^{\mathrm{a}, \mathrm{b}}(3,00 / 6,50)$ & \\
G4 & $10,00(6,00 / 11,75)$ & $3,00(2,00 / 4,50)$ & $6,00^{\mathrm{a}, \mathrm{b}}(3,25 / 7,75)$ & \\
\hline
\end{tabular}

Mediana (Quartil 25 / Quartil 75) da cor inicial, final e resultado do tratamento (após 7 dias). Letras iguais indicam ausência de diferença estatisticamente significativa.

\section{Discussão}

$\mathrm{Na}$ presente pesquisa avaliamos a modificação de protocolo através dos aparelhos emissores de luz halógena e LED. A aplicação de luz halógena associada ao protocolo proposto pelo fabricante obteve o menor clareamento dos dentes quando comparado aos resultados da aplicação única do peróxido (45 minutos). Este fato denota que, caso o profissional opte por utilizar tal fonte de luz, ele deve aplicar o peróxido em passo único. Contudo, não houve diferença estatisticamente significativa entre os grupos com diferentes fontes luminosas.

$\mathrm{Na}$ verdade, há dúvidas dos profissionais da Odontologia quanto a ecessidade, eficácia e finalidade dos aparelhos fotoativadores para ativação dos gentes clareadores durante os procedimentos de clareamento dental. Na literatura 
existe certa divergência de opiniões em relação à modificação do protocolo de clareamento, por meio das fontes luminosas ${ }^{12}$.

Alguns autores condenam o uso de fonte luminosa, por afirmarem que a utilização dessas fontes pode gerar um calor capaz de lesar permanentemente os tecidos pulpares. Ainda destacam que não há qualquer eficácia na alteração de cor para utilização dessas luzes no resultado final do clareamento ${ }^{1,5-8}$. Fato esse também observado em nosso estudo.

Estudos realizados in vivo em dentes de humanos observou que o uso de luz acarretou uma perturbação da camada odontoblástica no tecido pulpar de pré-molares após o tratamento clareador de consultório ${ }^{13}$. Além disso, outro estudo observou a formação de áreas de necrose de coagulação em incisivos inferiores submetidos ao tratamento clareador ${ }^{14}$.

As lâmpadas halógenas, arco de plasma e outras lâmpadas emitem curtos comprimentos de onda tão bem quanto às ondas infravermelhas invisíveis e térmicas (750nm a $1 \mathrm{~mm})$ com pouca energia de fótons, promovendo aquecimento, que é desvantajoso no clareamento dentário ${ }^{15}$. Os aparelhos de luz halógena apresentam vantagens como tecnologia de baixo custo, boa intensidade de potência e emitem luz num espectro mais amplo. Porém algumas desvantagens também estão presentes como requererem manutenção, geram altas temperaturas e possuírem filtros redutores $^{16}$. O uso das luzes halógenas, por si, quando empregadas na ativação do peróxido de hidrogênio a 35\%, não traz qualquer benefício para o dentista ou o paciente. Não havendo qualquer necessidade de aplicação da mesma no procedimento de clareamento ${ }^{17}$.

De maneira semelhante ao proposto em nosso estudo, os resultados encontrados por Liang et al (2012) indicam que o clareamento eficaz dos dentes seria alcançado com um agente clareador altamente concentrado, com ou sem ativação de qualquer fonte de luminosa ${ }^{18}$.

Vários estudos demonstraram que a luz teve pouca influência sobre a resposta clareamento ao longo do período de observação. Os autores concluíram que o tema de clareamento em consultório, seja ele com ou sem fotoativação se mostra 
bastante eficaz. O envolvimento da luz halógena pode aumentar o efeito do clareamento imediato, porém, ele mostra pouca influência sobre o efeito do clareamento a longo prazo $^{1,5-8}$. Isto é corroborado com o resultado do presente estudo, uma vez que o gel clareador por mais tempo se demonstrou mais efetivo, e o resultado independe da luz. Entretanto, o uso da luz pode ser nocivo aos pacientes, uma vez que sua utilização aponta para danos pulpares ${ }^{13,14}$.

O uso dos LED no clareamento dental surgiu após o emprego dos LED na fotopolimerização de resinas. Observando-se que, o fotopolimerizador tem um comprimento de onda menor que não gerava calor durante a fotopolimerização das resinas, o mesmo passou a ser uma opção empregada na técnica de clareamento ${ }^{19}$.

Autores afirmam que os sistemas LED se baseiam na conversão da energia luminosa em térmica, ao nível do gel clareador aplicado sobre as superfícies dentárias $^{19}$. Este processo aumenta as vibrações moleculares, promovendo assim um aumento na formação de radicais livres hidroxila, de modo a acelerar o processo de clareamento sem comprometer a subida da temperatura pulpar ${ }^{20-23}$. Os LED apresentam diversas vantagens, dentre essas pode-se citar a menor utilização de energia elétrica, custo acessível, a não indução do aumento da temperatura da estrutura dentária, além da associação com laser terapêutico no controle da hipersensibilidade 22 .

Apesar de controverso, estudos defendem a utilização de luzes durante o procedimento de clareamento. Para esses autores, as fontes luminosas aceleram a reação de catabolização do peróxido, levando a uma diminuição no tempo final do procedimento, sem que haja qualquer dano aos tecidos dentários desde que corretamente aplicado ${ }^{4,20-25}$. Nesse sentido, cabe ressaltar que existem diferentes técnicas e aparelhos empregados para realizar a modificação no protocolo de clareamento por meio das fontes luminosas. Pode-se obter modificações através da variação de tempo e intensidade da aplicação da luz e também quanto ao espectro de z empregado em diferentes aparelhos. Nesse sentido, Araújo et al (2009) demonstrou o tipo de luz empregada (espectro) pode interferir no clareamento ${ }^{25}$. 
No estudo com enfoques laboratoriais (in vitro) e clínicos (in vivo), verificou-se que os diferentes tipos de fonte de luz não melhoravam a efetividade do agente clareador a base de peróxido de hidrogênio, uma vez que, ao final dos tratamentos, todas as terapias apresentavam resultados bastante semelhantes, independentemente do uso ou não de alguma fonte luminosa. Isso comprova que, as fontes luminosas são de modo geral, dispensáveis para as técnicas de clareamento a base de peróxido de hidrogênio ${ }^{8}$.

Uma das limitações encontradas no presente estudo diz respeito a falta de material preciso (espectrofotômetro) para seleção precisa da cor, fato este minimizado com o treinamento e calibração dos pesquisadores quanto ao processo de seleção de cor. Além disso, até o presente momento, não foram encontrados na literatura estudos que avaliassem a influência de diferentes fontes luminosas no clareamento dentário quando há modificação dos protocolos de aplicação dos peróxidos.

\section{Conclusões}

Baseando-se nos resultados alcançados na pesquisa, tendo em vista as condições experimentais deste estudo, pode-se concluir que as modificações realizadas nos protocolos de clareamento, foram efetivas e satisfatórias para todos os grupos. Bem como, observa-se que na modificação do protocolo, em que o agente clareador é mantido na superfície dentária durante todo o tempo estimado, os resultados são mais satisfatórios.

Tendo em vista os resultados e a discussão encontrados na pesquisa, podemos verificar que as evidências científicas nos levam a observar que não há bases suficientes que comprovem a eficácia e benefícios a curto prazo da modificação dos protocolos de clareamento dental pelas fontes luminosas.

\section{Referências}

Marson FC, Sensi LG, Vieira LCC, Araújo E. Clinical Evaluation of In-office ntal Bleaching Treatments With an Without the Use of Light-activation Sources. er Dent. 2008;33(1):15-22.

Mondelli RFL. Comparative clinical study of the effectiveness of different tal bleaching methods - two year follow-u.p. J Appl Oral Sci. 2012;20(4):435-43. 
3. Gallinari MO. Analysis of the bleaching efficacy and adverse effects caused by the use of violet light in dental bleaching [Tese]. Araçatuba: Faculdade de Odontologia/Universidade Estadual Paulista; 2019.

4. Luk K, Tam L, Hubert M. Effect of light energy on peroxide tooth bleaching. J Am Dent Assoc. 2004;135(2):194-201.

5. Buchalla W, Attin T. External bleaching therapy with activation by heat, light or laser-a systematic review. Dent Mater. 2007;23(5):586-96.

6. Polydorou O, Wirsching M, Wokewitz M, Hahn P. Three-month evaluation of vital tooth bleaching using light units: A randomized clinical study. Oper Dent. 2013;38(1):21-32.

7. Klaric E, Rakic M, Sever I, Tarle Z. Temperature rise during experimental lightactivated bleaching. Lasers Med Sci. 2015;30(2):567-76.

8. Almeida LCAG, Riehl H, dos Santos PH; Sundfeld MLMM; Briso ALF. Clinical evaluation of the effectiveness of different bleaching therapies in vital teeth. Int $\mathrm{J}$ Periodontics Restorative Dent, 2012;32(3):303-09.

9. Moncada G, Sepúlveda D, Elphick K, Contente M, Estay J, Bahamondes V, et al. Effects of Light Activation, Agent Concentration, and Tooth Thickness on Dental Sensitivity After Bleaching. Oper Dent. 2013;38(5):467-76.

10. Santos TRB, Pereira RGS, Alves PVM, Gomes TG, Sette-de-Souza PH. Avaliação de diferentes protocolos no clareamento dentário. Arch Health Invest. 2018;7(10):42529.

11. Goncalves MLL. In-Office Tooth Bleaching for Adolescents Using Hydrogen Peroxide-Based Gels: Clinical Trial. Braz. Dent. J. 2017;28(6):720-25.

12. Benetti F, Lemos CAA, Gallinari MO, Terayama AM, Briso ALF, Jacinto RC, et al. Influence of different types of light on the response of the pulp tissue in dental bleaching: a systematic review. Clin Oral Investig. 2018;22(4):1825-37.

13. Marson FC, Gonçalves RS, Silva CO, Cintra LTÂ, Pascotto RC, dos Santos PH, et al. Penetration of hydrogen peroxide and degradation rate of different bleaching products. Oper Dent. 2015;40(1):72-9.

14. Florez FLE, Andrade MF, Campos EA, Oliveira Júnior OB, Bagnato VS, Panhoca $\mathrm{VH}$. In-Office dental bleaching efficacy assessment in function of the light exposure gime by digital colometric reflectance spectroscopy. J Dent Oral Hyg. 2011;3(8):99105 .

Carrasco, LD. Avaliação da eficácia e do aumento da permeabilidade tinária, no clareamento dental interno, após a utilização de peróxido de hidrogênio 
a 35\% com ativação por led, luz halógena e na técnica "walking bleach' [Dissertação]. Ribeirão Preto: Faculdade de Odontologia de Ribeirão Preto/USP; 2004.

16. Machado CT, Seabra BGM, Santos AJS, Hippolito MP, Lanverly BCS. Novos Tipos de Fontes Fotopolimerizadoras Existentes no Mercado: Conceitos Atuais. Odontol. Clin.-Cient. 2007;6(3):207-11.

17. Papathanasiou A, Kastali S, Perry RD. Clinical evaluation of a 35\% hydrogen peroxide in-office whitening system. Compend Contin Educ Dent. 2002;23(4):33-58.

18. Liang S, Sa Y, Sun L, Ma X, Wang Z, Xing W, et al. Effect of halogen light irradiation on hydrogen peroxide bleaching: an in vitro study. Aust Dent J. 2012;57(3):277-83.

19. Millis RW. Blue light emitting diodes- an alternative method of light curing? Br Dent J. 1995;32(8):178-69.

20. Zanin F, Brugnera Junior A, Bassoukou HI. Dental bleaching with laser and LEDs. In: Laser In Dentistry Practical Dental Book. M. Vitale, C. Caprioglio (eds.). Bologna: Martina; 2010, p.57-73.

21. Hahn P, Schondelmaier N, Wolkevitz M, Altenburger MJ, Polydorou O. Efficacy of tooth bleaching with and without light activation and its effect on the pulp temperature: an in vitro study. Odontology. 2013;101(1): 67-74.

22. Riehl H, Nunes MF. As fontes de energia luminosa são necessárias na terapia de clareamento dental? CIOSP; 2007, p.200-32.

23. Bortolatto JF, Pretel H, Neto CS, Andrade MF, Moncada G, Oliveira Junior OB. Effects of LED-laser hybrid light on bleaching effectiveness and tooth sensitivity: a randomized clinical study. Laser Phys Lett. 2013;10(8):560-81.

24. Tavares M, Stultz J, Newman M, Smith V, Kent R, Carpino E, et al. Light augments tooth whitening with peroxide. J Am Dent Assoc. 2003;134(2):167- 75.

25. Araújo $R$, Torres $C$, Araújo $M$. In vitro evaluation of dental bleaching effectiveness using hybrid lights activation. Rev Odonto Ciênc. 2009;25(2):159-64. 